\title{
Compact, Low-cost GNSS Modules for Precise Point Positioning
}

\author{
Anindya Bose*, Somnath Mahato, Sukabya Dan and Atanu Santra \\ Department of Physics, The University of Burdwan, Golapbag, Burdwan 713 104, India
}

\begin{abstract}
Global Navigation Satellite System (GNSS) uses Precise Point Positioning (PPP) technique to find out accurate geolocation information of any point. Generally, costly, geodetic GNSS receivers are used for PPP. This manuscript presents the results of studies on the usability of commercial, compact, cost-effective GNSS modules with commercial antennas for PPP in comparison to commonly used geodetic, costly receivers from India, which is a excellent location for GNSS use. Compact GNSS modules from two manufacturers are used in the study, and the encouraging results show the clear advantage of cost, size, and power requirements of such modules. The modules provide sub-cm horizontal solution accuracy which is very similar to those obtained using geodetic receivers, and around $20 \mathrm{~cm}$ accuracy in the vertical coordinate, which is slightly inferior to the results provided by the geodetic reveivers. Results of this novel study would be useful for implementing cost-efficient GNSS PPP in real life, in highly demanding geodetic applications including CORS establishment and PPP.
\end{abstract}

\section{Introduction}

Precise Point Positioning (PPP) is an important method in Global Navigation Satellite System (GNSS) technology for providing extremely accurate position solutions. PPP is used for highly demanding GNSS applications like surveying and other geodetic applications [1,2]. Using PPP, the GNSS users may obtain solution accuracy to the order of few cms and the method is applicable for any point on the globe as this standalone method does not has baseline restrictions. The basic concept of PPP lies in carrier phase data processing along with the use of data products from the International GNSS Service (IGS) [3, 4]. With the popularity of the GNSS Real Time Kinematic (RTK) technique, PPP is used for the establishment of RTK Base station at a precisely known location, and also for the establishment of Continuously Operating Reference Stations (CORS).

Currently, multiple global (GPS, GLONASS, Galileo, Beidou) and regional (QZSS and NavIC) components of GNSS are operational; as of mid-August 2021, a total of 144 navigation satellites are in the orbit, out of which 129 are in operation [5-8]. India is geographically situated in a favorable location to receive signals from all satellite constellations. It is noted that around 50 navigation satellites are always visible from any part

\footnotetext{
* Corresponding author: abose@ phys.buruniv.ac.in
} 
of India [9] and therefore, India is a good location for GNSS utilization, and studies on PPP are interesting aspects for enhancing the applications of GNSS.

Generally, costly, geodetic grade, multi-constellation, multi-frequency GNSS receivers are used for GNSS-PPP purposes. With the advancement of GNSS hardware technology, compact, low-cost GNSS modules are commercially available that are predominantly used for mass-market commercial applications. In this manuscript, results from India on GNSS PPP using these compact, cost-efficient modules are presented which is a novel effort using such modules vis-à-vis the performance of geodetic receivers. Applicability of such modules for GNSS PPP will pave the way for cost, size, and power-efficient utilization of GNSS for precise positioning applications including RTK. The manuscript is organized in the following manner: Section 2 presents a brief discussion on GNSS PPP, Section 3 presents the background experience of GNSS PPP from India using geodetic receivers, Section 4 presents the detailed results on GNSS PPP using two compact GNSS modules and finally, the conclusions are presented in section 5 .

\section{GNSS PPP: A brief discussion}

PPP is a post-processing method to obtain point positioning accuracy by using un-differenced observables obtained from single geodetic grade GNSS receivers. PPP uses precise ephemerides, clock files, bias and atmospheric effects corrections [10]. Online PPP service has become an important means for GNSS users for a different perspective of applications.

AUSPOS, an online GPS data processing service, provides a precise position solution [11]; this service is offered by Australian Geoscience. Though it does not follow the conventional PPP technique, it can provide a highly accurate position solution and can be used in many geodetic applications. The backbone of this service is Bernese Software. The computation process uses a relative positioning method where the nearest 15 IGS stations are used as references, Earth Orientation Parameters (EOP) and IGS products. Precise solution using the double-difference technique is computed using these 15 stations [12]. It relies on the baseline processing method. A double difference of ionosphere-free linear combination of RINEX observables is used in this computing process.

Canadian Spatial Reference System-PPP (CSRS-PPP) provides a precise position solution and uses the conventional PPP method. The Geodetic Survey Division (GDS) of Natural Resources Canada (NRCan) introduced it in 2003 [10] and it uses precise satellite orbit, clock, and bias corrections derived from the global IGS network [13]. The output includes station position, station clock status, tropospheric zenith delay, and carrier phase ambiguities [10].

AUSPOS requires only GPS RINEX observables whereas the NRCan CSRS-PPP service can compute on standalone GPS, GLONASS, and GPS+GLONASS RINEX data as well. AUSPOS do not compute on GLONASS till now.

As these processing services provide accurate and precise position solutions, the solutions provided by the services can be used for creation of permanent reference stations for Network Transfer of RTCM messages through Internet Protocol (NTRIP) services, in deformation and crustal movement monitoring, for site selection in case of mounting Continuously Operating Reference Station (CORS) in the vicinity of densely populated multipath prone areas [14].

\section{GNSS PPP using geodetic receivers at GLB, India}

To initiate the study, results of a previous effort to use geodetic, multi-constellation, multifrequency geodetic GNSS receivers for GNSS PPP from GNSS Laboratory, The University 
of Burdwan, Indian (GLB) is presented [15]. This work forms the basis for understanding the capability of the compact modules for PPP in comparison to the geodetic grade receivers. In the effort, 2 GNSS geodetic receivers were used for PPP. Firstly, a Leica GR50 geodetic receiver with a Leica AR25 Choke ring antenna (total cost USD 22,000) was used at location \#1. Receiver Independent Exchange Format (RINEX 2.11) GPS-only data is collected for 24 hours $@ 1 \mathrm{~Hz}$ on April 12, 2019, and the data is uploaded to the AUSPOS online PPP processing service of geoscience Australia [11]. The PPP solution quality results obtained from the AUSPOS service are shown in Table 1.

Table 1. Results for GPS-only PPP for GLB, India (Location \#1) using Leica GR50 receiver (AUSPOS online service, Geoscience Australia)

\begin{tabular}{|c|c|c|c|}
\hline \multirow{2}{*}{ Location id } & \multicolumn{3}{|c|}{ Position Uncertainty (95\% confidence level), Geodetic ITRF 2014, m } \\
\cline { 2 - 4 } & Latitude (East) & Longitude (North) & Ellipsoid Height (Up) \\
\hline$\# 1$ & 0.006 & 0.004 & 0.010 \\
\hline
\end{tabular}

In the second effort, another geodetic receiver, Javad DELTA G3T is used with a Javad GrAnt G3T geodetic antenna (total cost USD 11,000) at location \#2 of GLB, India to collect GPS+GLONASS data as shown in Figure 1. Raw data (.jps format) collected @1 $1 \mathrm{~Hz}$ for 24 hours each is converted to RINEX 2.11 format and uploaded to NRCan online PPP service from Natural Resources, Canada [13]. The position uncertainty (95\%) for consecutive 7 days in 2019 in concurrent GPS, GLONASS, and GPS+GLONASS operation as obtained from the online PPP service is shown in Table 2 [15].

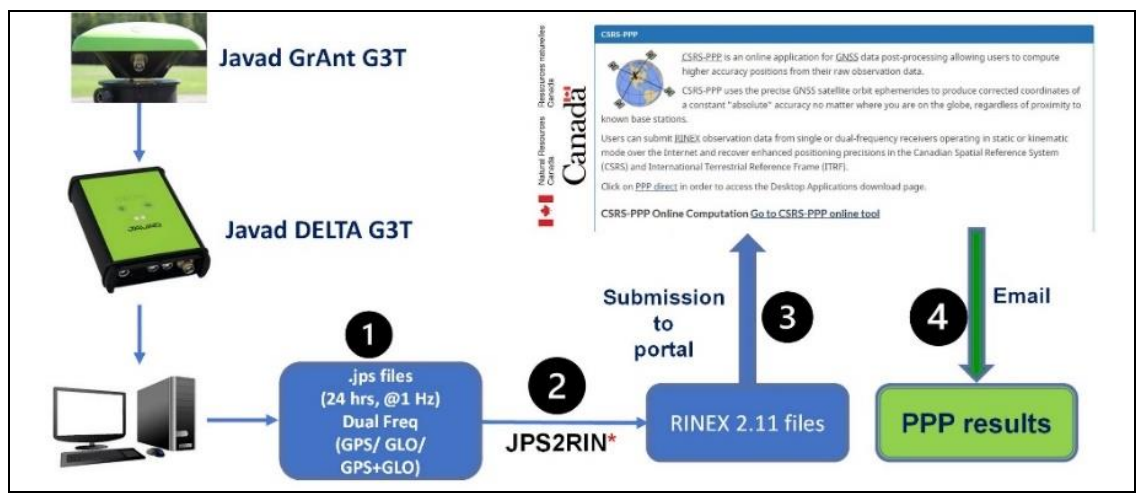

Fig. 1. Experimental setup for studying PPP performance using geodetic receiver at GLB, India (Location \#2).

Table 2. Results for GPS, GLONASS, and GPS+GLONASS PPP for GLB, India (Location id \#2) using Javad DELTA G3T receiver (NRCan online service, Natural Resources Canada)

\begin{tabular}{|c|c|c|c|c|c|c|c|c|c|}
\hline \multirow{2}{*}{ Date } & \multicolumn{6}{|c|}{ Position Uncertainty (95\% confidence level), Geodetic ITRF 2014, $\mathbf{~ m}$} \\
\cline { 2 - 10 } & \multicolumn{2}{|c|}{ Latitude (East) } & \multicolumn{2}{c|}{ Longitude (North) } & \multicolumn{3}{c|}{ Ellipsoid Height (Up) } \\
\cline { 2 - 9 } & GPS & GLO & $\begin{array}{c}\text { GPS+ } \\
\text { GLO }\end{array}$ & GPS & GLO & $\begin{array}{c}\text { GPS+ } \\
\text { GLO }\end{array}$ & GPS & GLO & $\begin{array}{c}\text { GPS+ } \\
\text { GLO }\end{array}$ \\
\hline $17 / 12 / 2019$ & 0.004 & 0.009 & 0.004 & 0.007 & 0.013 & 0.007 & 0.015 & 0.033 & 0.015 \\
\hline $18 / 12 / 2019$ & 0.003 & 0.004 & 0.003 & 0.009 & 0.008 & 0.006 & 0.013 & 0.019 & 0.010 \\
\hline
\end{tabular}




\begin{tabular}{|l|l|l|l|l|l|l|l|l|l|}
\hline $19 / 12 / 2019$ & 0.003 & 0.005 & 0.003 & 0.008 & 0.009 & 0.006 & 0.014 & 0.021 & 0.011 \\
\hline $20 / 12 / 2019$ & 0.003 & 0.004 & 0.002 & 0.008 & 0.008 & 0.005 & 0.013 & 0.018 & 0.010 \\
\hline $21 / 12 / 2019$ & 0.003 & 0.004 & 0.002 & 0.009 & 0.008 & 0.005 & 0.013 & 0.016 & 0.010 \\
\hline $22 / 12 / 2019$ & 0.003 & 0.004 & 0.002 & 0.008 & 0.008 & 0.005 & 0.013 & 0.017 & 0.010 \\
\hline $23 / 12 / 2019$ & 0.003 & 0.004 & 0.002 & 0.008 & 0.007 & 0.005 & 0.012 & 0.017 & 0.010 \\
\hline
\end{tabular}

Tables 1 and 2 provide a clear idea about the quality of GNSS-PPP position solution using geodetic GNSS receivers; it is also witnessed that GPS+GLONASS mode of operation provides better PPP solution quality. These results serves as the background for the subsequent study and would be useful in understanding the usability of the compact, lowcost GNSS modules for PPP which is presented in the next section.

\section{GNSS PPP using compact modules at GLB, India}

In this endeavor, two compact, low-cost, multi-constellation, multi-frequency, carrier-phase enabled GNSS modules are used for PPP positioning to study the usability of such modules for GNSS PPP purpose in the background of the performance of the geodetic receivers.

\subsection{GNSS Modules}

A detailed description of the two compact GNSS evaluation board (EVB) modules, viz. uBLOX ZED F9P and NTLab NTL104, used for the study is shown in Table 3.

Table 3. Compact GNSS modules used for PPP at GLB, India.

\begin{tabular}{|c|c|c|c|c|c|}
\hline $\begin{array}{l}\text { Make and } \\
\text { Model, } \\
\text { (No of } \\
\text { Channels) }\end{array}$ & Picture & $\begin{array}{l}\text { Constellations } \\
\text { Supported }\end{array}$ & $\begin{array}{c}\text { Frequency of } \\
\text { operation }\end{array}$ & $\begin{array}{c}\text { EVB size } \\
\text { and } \\
\text { (List } \\
\text { Price) }\end{array}$ & Link \\
\hline $\begin{array}{c}\text { uBLOX } \\
\text { ZED F9P } \\
\text { (184) }\end{array}$ & & $\begin{array}{c}\text { GPS, } \\
\text { GLONASS, } \\
\text { Galileo, Beidou, } \\
\text { QZSS, SBAS }\end{array}$ & $\begin{array}{c}\text { GPS L1, L2 } \\
\text { GLONASS L1, L2 } \\
\text { Galileo E1, E5 } \\
\text { Beidou B1, B2 }\end{array}$ & $\begin{array}{c}4.7 \times 3.2 \\
\text { cm } \\
\text { (Euro } \\
209.99)\end{array}$ & $\begin{array}{l}{[16,} \\
17]\end{array}$ \\
\hline $\begin{array}{c}\text { NTLAB } \\
\text { NTL104 } \\
(128)\end{array}$ & & $\begin{array}{c}\text { GPS } \\
\text { GLONASS } \\
\text { Galileo, Beidou } \\
\text { QZSS, SBAS, } \\
\text { NavIC }\end{array}$ & $\begin{array}{c}\text { GPS L1, L2/ L5 } \\
\text { GLONASS L1, L2 } \\
\text { Galileo E1, E5a/ E5b } \\
\text { Beidou B1, B2 } \\
\text { NavIC L5, S }\end{array}$ & $\begin{array}{c}7.0 \times 4.5 \\
\mathrm{~cm} \\
(\mathrm{USD} \\
850.00)\end{array}$ & [18] \\
\hline
\end{tabular}

\subsection{Data Collection Method}

For data collection, the uBLOX ZED F9P module is used together with a uBLOX ANN Multiband commercial GNSS patch antenna [19] as shown in Figure 2. The GNSS module is connected to the USB port of a computer that also supplies the power to the module. For a complete day in February 2021, raw dual-frequency GPS data (.ubx format) is collected @1 $\mathrm{Hz}$. The raw data is converted to RINEX 2.11 format using RTKLib, an open-source GNSS data processing software [20]. The RINEX data is then uploaded to AUPOS online GPS processing service from Geoscience Australia [11]. The PPP solution results are received back by email. The data collection and processing schematic are shown in Figure 2. 


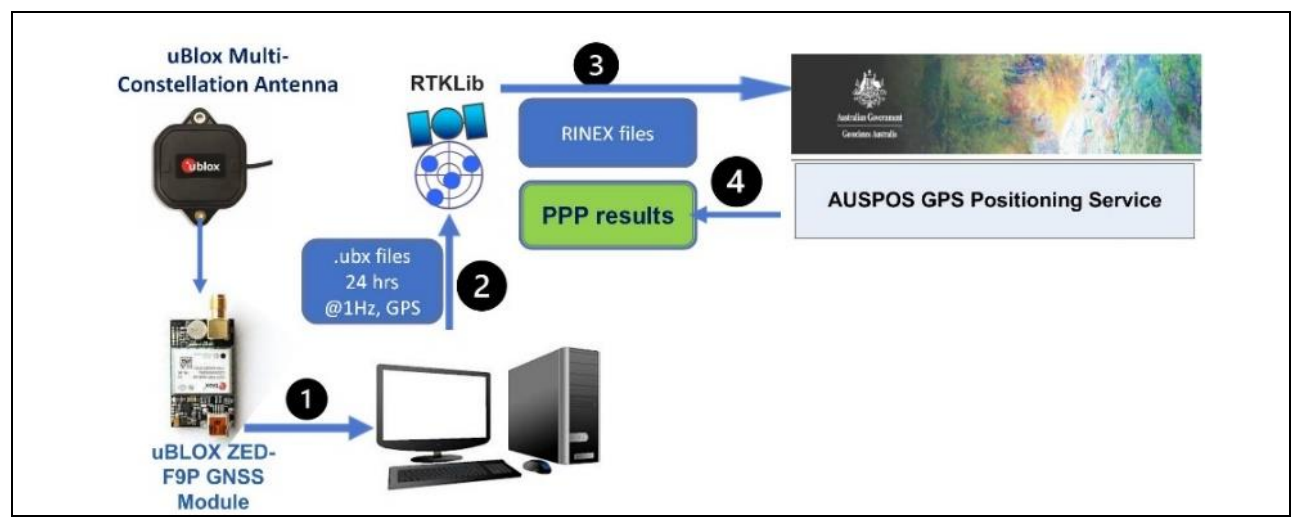

Fig. 2. Experimental setup for studying PPP performance of ZED-F9P module at GLB, India

For data collection using the NTL104 module, the same is used similarly with the uBLOX Multiband patch antenna as shown in Figure 3. The NTL104 module is connected to a computer through the USB port that also supplies the power to the module. For consecutive 3 days in April 2021, dual-frequency GPS, GLONASS, and GPS+GLONASS RINEX 2.11 data are collected @1 Hz. The RINEX data is uploaded to NRCan online GNSS processing service of Natural Resources, Canada [13]. The PPP solution results are received back by email. The data collection and processing schematic are shown in Figure 3.

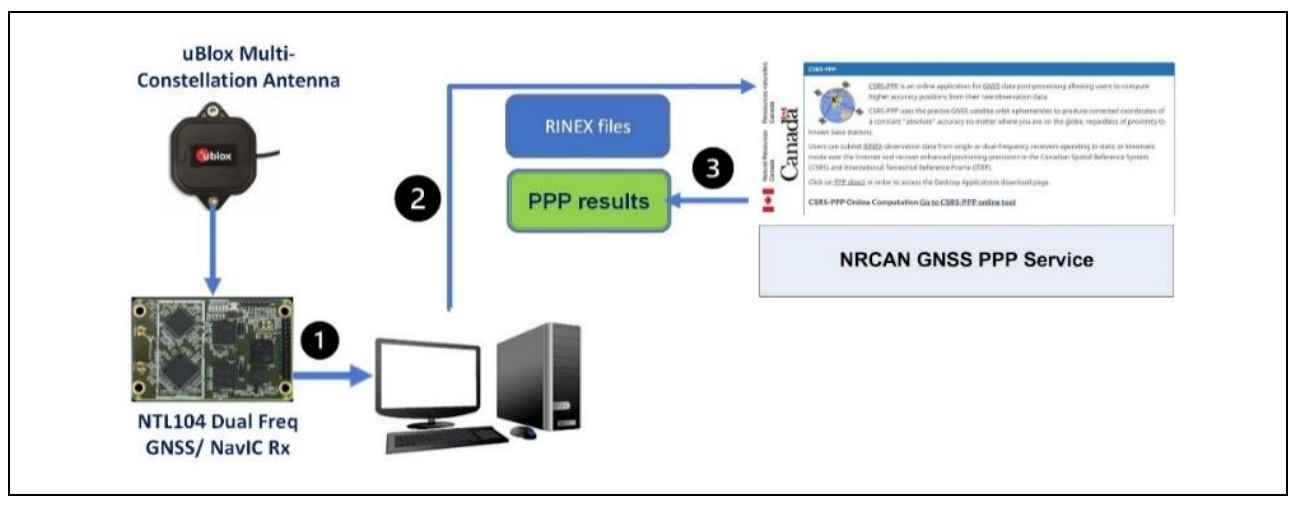

Fig. 3. Experimental setup for studying PPP performance of NTL 104 module at GLB, India

\subsection{Results and discussions}

The PPP results (GPS-only results for ZED F9P module and GPS, GLONASS, and GPS+GLONASS results) as obtained from the online PPP service are shown in Table 4. For both the compact modules used in the study, it is witnessed that, below $5 \mathrm{~mm}$ horizontal position accuracy is achievable in GPS or GPS+GLONASS hybrid mode of operation with a $95 \%$ confidence level. In the vertical coordinate, the achievable accuracy level is below 20 $\mathrm{cm}$.

Now, the PPP performance of the compact modules shown in Table 4 are compared with those for the geodetic GNSS receivers as presented in Tables 1 and 2. It may be noted that the positioning performance is similar for the horizontal coordinates, and the vertical PPP accuracy provided by the compact modules are slightly inferior to their geodetic counterpart. If the cost, size, and power requirements for the two types of GNSS receivers are compared, it may be seen that the compact GNSS modules have clear advantage of much lower cost, 
lower size and weight; the compact modules also consume very low power. Therefore, with similar or slightly inferior performances and advantages from practical viewpoints, the compact GNSS modules are potential candidates for PPP.

Table 4. Results for GNSS PPP for GLB, India using compact GNSS modules

\begin{tabular}{|c|c|c|c|c|c|c|c|c|c|}
\hline \multirow{3}{*}{$\begin{array}{l}\text { Module Used } \\
\text { (Date) }\end{array}$} & \multicolumn{9}{|c|}{ Position Uncertainty (95\% confidence level), Geodetic ITRF 2014, m } \\
\hline & \multicolumn{3}{|c|}{ Latitude (East) } & \multicolumn{3}{|c|}{ Longitude (North) } & \multicolumn{3}{|c|}{ Ellipsoid Height (Up) } \\
\hline & GPS & GLO & $\begin{array}{l}\text { GPS+ } \\
\text { GLO }\end{array}$ & GPS & GLO & $\begin{array}{l}\text { GPS+ } \\
\text { GLO }\end{array}$ & GPS & GLO & $\begin{array}{c}\text { GPS } \\
+\mathrm{GL} \\
\mathrm{O}\end{array}$ \\
\hline $\begin{array}{c}\text { ZED-F9P } \\
\text { (Feb. 2021) }\end{array}$ & 0.005 & - & - & 0.003 & - & - & 0.010 & - & - \\
\hline $\begin{array}{c}\text { NTL 104 } \\
(24 / 04 / 2021)\end{array}$ & 0.003 & 0.080 & 0.003 & 0.003 & 0.015 & 0.003 & 0.013 & 0.036 & 0.012 \\
\hline $\begin{array}{c}\text { NTL 104 } \\
(25 / 04 / 2021) \\
\end{array}$ & 0.003 & 0.013 & 0.003 & 0.003 & 0.025 & 0.003 & 0.013 & 0.059 & 0.013 \\
\hline $\begin{array}{c}\text { NTL 104 } \\
(26 / 04 / 2021) \\
\end{array}$ & 0.004 & 0.122 & 0.004 & 0.004 & 0.279 & 0.004 & 0.018 & 0.267 & 0.018 \\
\hline
\end{tabular}

\section{Conclusions}

In this work, the usability of commercial, compact, dual-frequency GNSS modules is studied for the GNSS PPP solution. With the background of the results using two geodetic receivers, PPP solution quality provided by compact modules from two manufacturers has been studied. Encouraging results show that with clear advantages of cost, size, and power requirements, the compact modules can be efficiently used for GNSS PPP. In GPS-only or GPS+GLONASS hybrid operation, the modules can be used for PPP with sub-cm accuracy in horizontal coordinates and below $20 \mathrm{~cm}$ accuracy in the vertical coordinate. Therefore, the modules are potentially good candidates for precise geodetic applications, as CORS stations and for GNSS RTK Base applications in a cost-efficient manner.

Future work would include tri and quad-constellation PPP using such modules and studies using similar dual-frequency, multi-constellation modules from other manufacturers. Considering the potential of the modules for GNSS RTK, future work would also involve the development of a cost-efficient NTRIP Caster service.

The authors acknowledge Integrated Test Range (ITR), DRDO, Chandipur, India and All India Council of Technical Education (AICTE), New Delhi India for financial support, and NTLab UAB, Lithuania for hardware support.

\section{References}

1. K. Mostafa. On the suitability of generalized regression neural networks for GNSS position time series prediction for geodetic applications in geodesy and geophysics. arXiv preprint arXiv:2005.11106 (2020).

2. P. D. Oluyori, M. N. Ono, S. O. Eteje. Computations of Geoid Undulation from Comparison of GNSS/Levelling with EGM 2008 for Geodetic Applications. International Journal of Scientific and Research Publications 8,10, 235-241, (2018).

3. M. Ge, G. Gendt, M. A. Rothacher, C. Shi, J. Liu. Resolution of GPS carrier-phase ambiguities in precise point positioning (PPP) with daily observations. Journal of geodesy, 82,7, 389-399, (2008). 
4. Y. Gao, X. Shen. A New Method for Carrier-Phase-Based Precise Point Positioning. Navigation, 49,2,109-116, (2002).

5. Applied Consumer Center GLONASS, Available via https://www.glonass-iac.ru/, Accessed on 17 August 2021.

6. Constellation Information, Available via https://www.gsc-europa.eu/system-servicestatus/constellation-information, Accessed on 19 August 2021.

7. S. Mahato, A.Santra, S. Dan, P. Verma, P. Banerjee, A. Bose, Visibility anomaly of GNSS satellite and support from regional systems. Current Science, 119,11, 1774-1782, (2020). DOI: 10.18520/cs/v119/i11/1774-1782

8. QZSS Satellite Information, Available via https://qzss.go.jp/en/technical/qzssinfo/index.html, Accessed on 17 August 2021.

9. M. Goswami, S. Mahato, R. Ghatak, A. Bose, Potential of Multi Constellation GNSS in Indian Missile Test Range Applications. Defence Science Journal, 70,6, 682-691, (2020). DOI: 10.14429/dsj.70.15570

10. Y. Mireault, P. Tétreault, F. Lahaye, P. Héroux, J. Kouba,. Online precise point positioning, GPS world, 19, 9, 59-64, (2008).

11. AUSPOS, Australian Geoscience, Available via http://www.ga.gov.au/scientifictopics/positioning-navigation/geodesy/auspos/faq1, Accessed on 28 August 2021.

12. AUSPOS-How it works, Available via https://www.ga.gov.au/scientifictopics/positioning-navigation/geodesy/auspos/faq3 Accesed on 28 August 2021.

13. Canadian Spatial Reference System Precise Point Positioning (CSRS-PPP), Geodetic tools and data, Available via https://www.nrcan.gc.ca/maps-tools-andpublications/tools/geodetic-reference-systems/data/10923, Accessed on 28 August 2021.

14. N. AbouAly, M. Elhussien, M. Rabah, Z. Zidan, Assessment of NRCAN PPP online service in determination of crustal velocity: case study Northern Egypt GNSS Network. Arabian Journal of Geosciences, 14, 3, 1-15, (2021).

15. A. Santra, A. Dutta, P. Banerjee, A. Bose, A Study on Multi-GNSS Precise Point Positioning from India, Proc. URSI GASS 2020, 29 August - 5 September 2020; Rome, (2020).

16. ZED-F9P module, u-blox F9 high precision GNSS module, Available via https://www.u-blox.com/en/product/zed-f9p-module, Accessed on 17 August 2021.

17. ZED-F9P RTK GNSS receiver board with SMA Base or Rover, Available via https://www.gnss.store/gnss-gps-modules/99-ublox-zed-f9p-rtk-gnss-receiver-boardwith-sma-base-or-rover.html, Accessed on 18 August 2021.

18. NTL104 Dual antenna high performance OEM GNSS module with S-band support RTK/PPP INS, Available via https://ntlab.lt/product/gnss-receiver-module-ntl104/, Accessed on 17 August 2021.

19. ANN-MB1 L1/L5 multi-band high precision GNSS antenna, Available via https://www.gnss.store/rf-gps-antennas/159-ublox-ann-mb-multi-band-high-precisiongnss-antennas.html, Accessed on 17 August 2021.

20. RTKLib 2.4.3, Available via http://www.rtklib.com/, Accessed on 13 october 2019. 